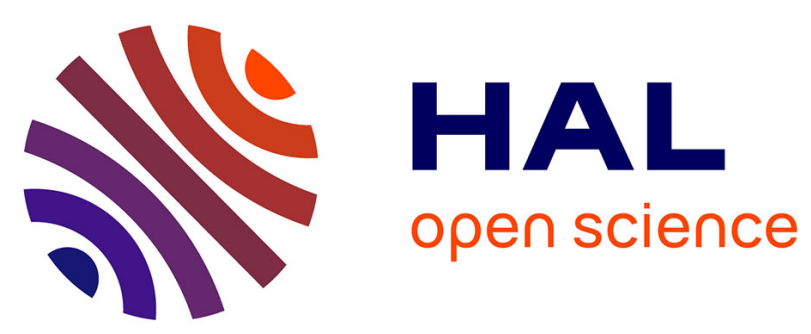

\title{
Effets d'irradiation sur l'état d'ordre chimique dans les alliages (Ni0.67Cr0.33)1-xFex
}

\author{
A. Frely, B. Beuneu, A. Barbu, G. Jaskierowicz
}

\section{To cite this version:}

A. Frely, B. Beuneu, A. Barbu, G. Jaskierowicz. Effets d'irradiation sur l'état d'ordre chimique dans les alliages (Ni0.67Cr0.33)1-xFex. Journal de Physique IV Proceedings, 1996, 06 (C2), pp.C2-109-C2-114. 10.1051/jp4:1996215 . jpa-00254193

\section{HAL Id: jpa-00254193 https://hal.science/jpa-00254193}

Submitted on 1 Jan 1996

HAL is a multi-disciplinary open access archive for the deposit and dissemination of scientific research documents, whether they are published or not. The documents may come from teaching and research institutions in France or abroad, or from public or private research centers.
L'archive ouverte pluridisciplinaire HAL, est destinée au dépôt et à la diffusion de documents scientifiques de niveau recherche, publiés ou non, émanant des établissements d'enseignement et de recherche français ou étrangers, des laboratoires publics ou privés. 


\title{
Effets d'irradiation sur l'état d'ordre chimique dans les alliages $\left(\mathrm{Ni}_{\mathbf{0 . 6 7}} \mathrm{Cr}_{\mathbf{0 . 3 3}}\right)_{1-\mathrm{x}} \mathrm{Fe}_{\mathrm{x}}$
}

\author{
A. Frely, B. Beuneu, A. Barbu et G. Jaskierowicz \\ Laboratoire des Solides Irradiés, Ecole Polytechnique, route de Palaiseau, 91128 Palaiseau cedex, France
}

\begin{abstract}
Résumé. L'effet de I'irradiation aux électrons à $400^{\circ} \mathrm{C}$ sur la mise en ordre à longue distance d'alliages $\left(\mathrm{Ni}_{0.67} \mathrm{Cr}_{0.33}\right)_{1-\mathrm{x}} \mathrm{Fe}_{\mathrm{x}}$ a été étudié par suivi de la résistivité électrique et par microscopie électronique en transmission pour les 3 compositions $x=0, x=0.05$ et $x=0.1$. Après irradiation, les pics de surstructure de la phase ordonnée de type $\mathrm{Pt}_{2} \mathrm{Mo}$ ont été observés dans tous les échantillons. La variation de résistivité électrique est semblable à celle observée durant un vieillissement thermique (état $\mathrm{K}$ ). La cinétique de mise en ordre à longue distance est fortement ralentie par l'addition de fer.
\end{abstract}

\begin{abstract}
The effect of $400^{\circ} \mathrm{C}$ electron irradiation on the long-range ordering of $\left(\mathrm{Ni}_{0.67} \mathrm{Cr}_{0.33}\right)_{1-\mathrm{x}} \mathrm{Fe}_{\mathrm{X}}$ alloys has been investigated by means of resistivity measurements and transmission electron microscopy for the 3 compositions $x=0, x=0.05$ and $x=0.1$. After irradiation, the Bragg peaks of the $\mathrm{Pt}_{2} \mathrm{Mo}$ type superstructure are observed for all samples. The variation of resisivity is very similar to that observed during thermal aging (K-state). The ordering reaction is strongly delayed by the addition of iron.
\end{abstract}

\section{INTRODUCTION}

Les alliages $\mathrm{Ni}-\mathrm{Cr}-\mathrm{Fe}$ autour de la composition $\mathrm{Ni}_{2} \mathrm{Cr}$ sont des matériaux qui possèdent des caractéristiques mécaniques élevées ainsi qu'une bonne résistance à la corrosion. Leurs propriétés mécaniques seraient cependant modifiées par une mise en ordre chimique, qui pourrait apparaître en particulier lorsque le matériau est utilisé sous irradiation.

En effet, des anomalies de résistivité et de chaleur spécifique [1][2] ont depuis longtemps permis de détecter une transition ordre-désordre dans les alliages nickel-chrome autour de la composition $\mathrm{Ni}_{2} \mathrm{Cr}$ lors de simples recuits thermiques.

L'alliage désordonné $\mathrm{Ni}_{2} \mathrm{Cr}$ est cubique à faces centrées, avec un paramètre cristallin $\mathrm{a}_{0} \cong 3,57 \AA$ proche de celui du nickel. La structure ordonnée a été déterminée par diffraction de rayons X [3], de neutrons [4] et d'électrons [5]. Elle est de type $\mathrm{Pt}_{2} \mathrm{Mo}$, du groupe Immm - $\mathrm{D}_{2 \mathrm{~h}}^{25}\left(\mathrm{n}^{\circ} 71\right)$. La maille est orthorhombique avec comme paramètres de maille $\mathrm{a}=3 \mathrm{a}_{0} / \sqrt{2}, \mathrm{~b}=\mathrm{a}_{0} / \sqrt{2}$ et $\mathrm{c}=\mathrm{a}_{0}$. Dans la direction (110) du CFC, il y a alternance de deux plans de nickel et d'un plan de chrome.

La température de transition $\mathrm{T}_{\mathrm{c}}$ se situe autour de $590^{\circ} \mathrm{C}$ [6]. La mise en ordre et la microstructure de la phase ordonnée ont été étudiés par suivi de la résistivité, des propriétés mécaniques et par microscopie électronique en transmission (voir [7-9] entre autres). Au cours d'un vieillissement au dessous de la température de transition, la résistivité, très sensible à l'état d'ordre, commence par augmenter, puis se stabilise pendant une période qui dépend de la température ( typiquement, une centaine d'heures à $500^{\circ} \mathrm{C}$ ). Elle chute ensuite lorsque l'ordre à longue distance (OLD) commence à s'établir.

La montée de résistivité est attribuée à une amplification de l'ordre à courte distance (OCD) ; l'état résultant, se maintenant tout au long du plateau, est connu sous le nom d' "état $K$ ".

Des études de vieillissement thermique [10] ont montré que l'addition de fer dans le système $\mathrm{Ni}-\mathrm{Cr}$ freine le processus de mise en ordre déjà très lent. A $500^{\circ} \mathrm{C}$, température optimale pour la mise en ordre, aucune mise en ordre n'a été constatée après deux années de maintien en température dans le cas de l'alliage contenant $10 \%$ at. de fer.

L'irradiation d'un tel alliage a, sur sa mise en ordre, deux effets opposés :

- en créant une forte sursaturation de lacunes et d'interstitiels, elle accélère la diffusion atomique et donc l'évolution vers l'état d'équilibre, qui peut être l'état ordonné,

- par déplacements atomiques (séquences de remplacement), elle crée du désordre. 
A très basse température le second effet est le seul opérant. Rivière et Eymery [11] l'ont observé en irradiant aux neutrons rapides à $20 \mathrm{~K}$ un échantillon de composition $\mathrm{Ni}_{0.67} \mathrm{Cr}_{0.33}$ dans différents états d'ordre initiaux : la résistivité des échantillons ordonnés à courte distance décroît tandis que celle des échantillons ordonnés à longue distance augmente, ce qui correspond dans les deux cas à une mise en désordre.

A plus haute température et à faible flux d'irradiation, inversement, l'effet d'accélération de la mobilité domine.

Nous présentons ici la première étude de l'effet de l'irradiation sur la mise en ordre dans des alliages $\left(\mathrm{Ni}_{0.67} \mathrm{Cr}_{0.33}\right)_{1-\mathrm{x}} \mathrm{Fe}_{\mathrm{x}}$ pour trois compositions : $\mathrm{x}=0,0.05$ et 0.1 (ce dernier alliage se rapprochant de l'alliage industriel Inconel 690) à une température de $400^{\circ} \mathrm{C}$.

\section{TECHNIQUES EXPERIMENTALES}

\subsection{Echantillons}

Ces alliages ont été élaborés par fusion par radiofréquences en lévitation magnétique à l'OM-ONERA de Châtillon. Les échantillons obtenus après découpe et polissage mécanique ont une taille standard (pour l'irradiation) de $30^{*} 10^{*} 0,3 \mathrm{~mm}^{3}$. Ils sont systématiquement recuits à $900^{\circ} \mathrm{C}$ pendant $30 \mathrm{mn}$ puis trempés à l'huile afin d'obtenir une solution solide homogène avec un faible taux de dislocations.

\subsection{Irradiations}

Les alliages modèles ont été irradiés avec des électrons de $2,5 \mathrm{MeV}$ jusqu'à des doses de $8.10^{19} \mathrm{e}^{-/ \mathrm{cm}^{2}}$ dans l'accélérateur Van de Graaff du Laboratoire des Solides Irradiés (Palaiseau). Le faisceau est balayé de façon à obtenir une irradiation homogène. Il est limité par un diaphragme de diamètre $20 \mathrm{~mm}$ placé devant

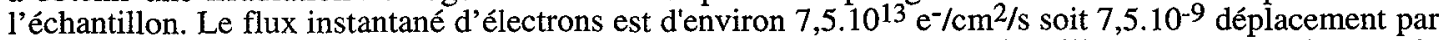
atome (d.p.a.) par seconde. Lors de l'irradiation, le chauffage de l'échantillon à une température de $400 \pm 5^{\circ} \mathrm{C}$ est assuré par la perte d'énergie des électrons qui le traversent. Les échantillons sont suspendus par les fils d'arrivée de courant utilisés pour les mesures de résistivité de façon à minimiser les pertes par conductions et donc les gradients de température au centre de l'échantillon.

Des mesures in situ de la résistivité électrique $(\rho)$ par la méthode des quatre points sont faites à intervalle régulier. L'espacement des prises de potentiel est de $4 \mathrm{~mm}$ de part et d'autre du centre de l'échantillon. Ces mesures sont effectuées hors faisceau à une température constante de $30^{\circ} \mathrm{C}$, ceci afin d'éliminer le bruit de fond lié aux fluctuations de température sous irradiation. L'incertitude relative sur les mesures de résistance est inférieure à $3 \cdot 10^{-5}$.

\subsection{Microscopie électronique}

Après irradiation, les zones irradiées des échantillons ont été analysées au microscope électronique en transmission sur le CM30 ( $300 \mathrm{KeV}$ ) du Laboratoire des Solides Irradiés. Pour cela, une pastille de $3 \mathrm{~mm}$ de diamètre est prélevée par carottage dans la région irradiée. Les pastilles sont ensuite amincies par polissage électrochimique dans une machine à double jet. La solution chimique employée est composée de $10 \%$ d'acide perchlorique, $20 \%$ d'éther monobutylique d'éthylène glycol et complétée par du méthanol (bain à $-30^{\circ} \mathrm{C}$, tension appliquée : $20 \mathrm{~V}$ ).

\section{RESULTATS}

\subsection{Résistivité électrique}

La figure 1 montre l'évolution de la résistivité électrique en fonction de la dose d'irradiation pour les 3 alliages : $\left(\mathrm{Ni}_{0.67} \mathrm{Cr}_{0.33}\right),\left(\mathrm{Ni}_{0.67} \mathrm{Cr}_{0.33}\right)_{0.95} \mathrm{Fe}_{0.05}$ et $\left(\mathrm{Ni}_{0.67} \mathrm{Cr}_{0.33}\right)_{0.90} \mathrm{Fe}_{0.10}$ (une dose de $10^{19} \mathrm{e}^{-} / \mathrm{cm}^{2}$ correspond à $10^{-3} \mathrm{dpa}$ et à un temps d'irradiation de 37 heures).

La résistivité électrique évolue de la même façon que lors des vieillissements thermiques mais il est manifeste que l'irradiation accélère fortement la mise en ordre.

On observe d'abord une augmentation (attribuée à l'état $\mathrm{K}$ pour les recuits thermiques). Ensuite, la résistivité électrique reste stable pendant une période d'autant plus longue que le pourcentage de fer est plus 
élevé. Pour l'alliage sans fer cette période n'est que de 10 heures à $400^{\circ} \mathrm{C}$ sous irradiation alors qu'elle durait une centaine d'heure hors irradiation à $500^{\circ} \mathrm{C}$. Enfin, la résistivité décroît du fait de la mise en ordre à longue distance. La variation de résistivité à saturation, tant absolu : que relative, semble d'autant plus faible que le pourcentage de fer est élevé.

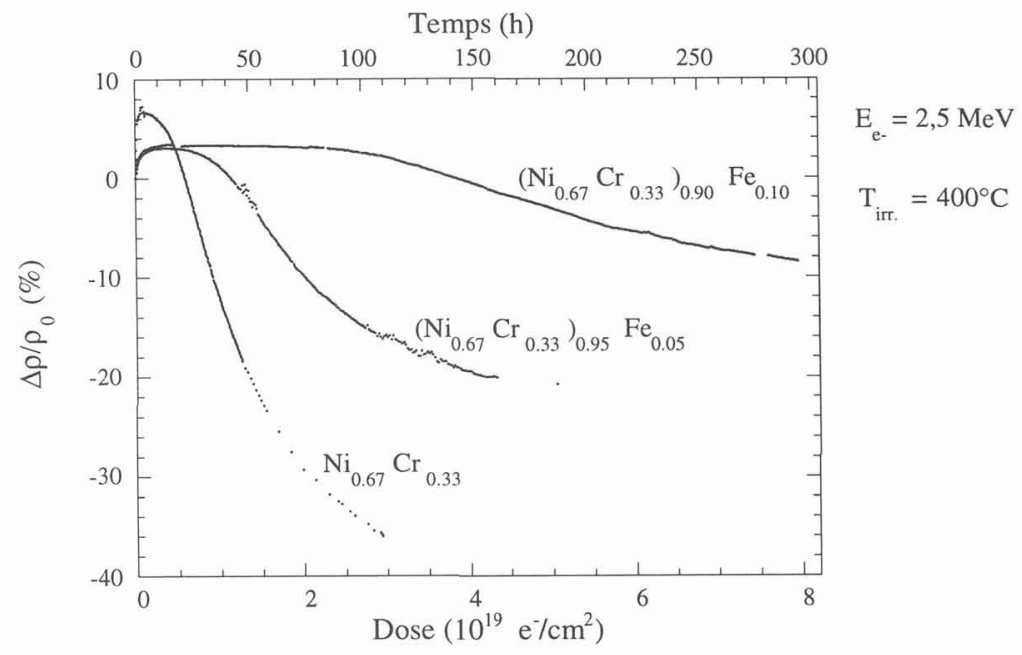

Figure 1 : Evolution de la mise en ordre dans $\left(\mathrm{Ni}_{0.67} \mathrm{Cr}_{0.33}\right)_{1-\mathrm{x}} \mathrm{Fe}_{\mathrm{x}}$ par mesure de résistivité électrique sous irradiation électronique.

\subsection{Microscopie électronique}

Les échantillons ont été observés en microscope électronique en transmission à la fin de l'irradiation. Les clichés de diffraction montrent que les taches de surstructure caractéristiques de la phase ordonnée, absentes avant irradiation, sont visibles quel que soit l'alliage irradié considéré (figure 2).

La surstructure présente 6 orientations possibles par rapport à la maille CFC, construites sur les 6 directions $\langle 110\rangle$. Les taches correspondant à ces 6 variants sont observées dans tous les cas.

L'intensité des taches de surstructure est qualitativement semblable pour les trois compositions. La microscopie ne nous donne pas d'information quantitative sur le degré d'ordre des échantillons.

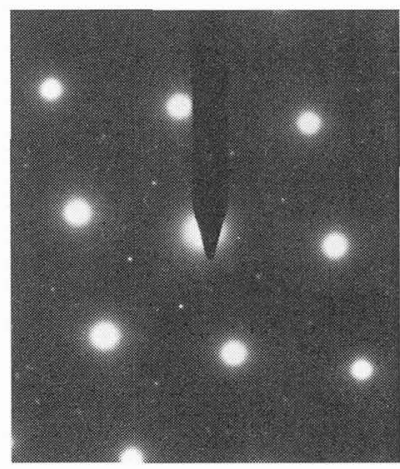

Nio.67 $\mathrm{Cr}_{0.33}-$ coupe $(110)$ cfc (fluence $=3.10^{19} \mathrm{e}^{-} / \mathrm{cm}^{2}$ )
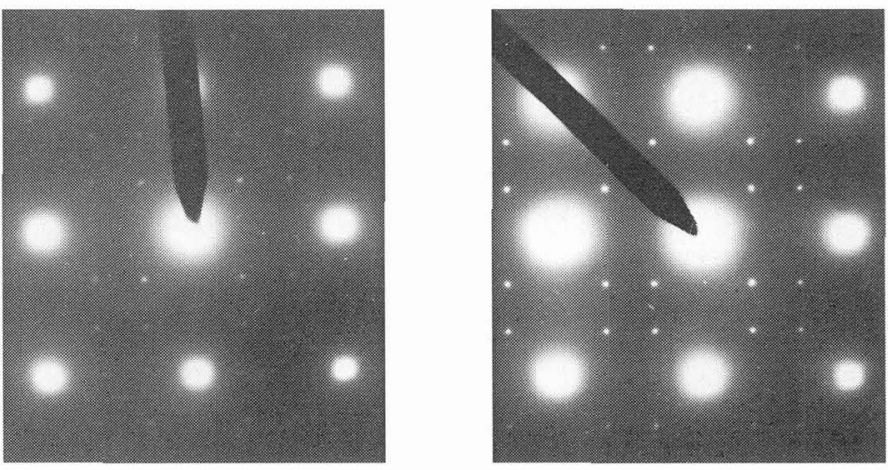

$\left(\mathrm{Ni}_{0.67} \mathrm{Cr}_{0.33}\right)_{0.95} \mathrm{Fe}_{0.05}$ - coupe $(001)_{\mathrm{cfc}}\left(\mathrm{Ni}_{0.67} \mathrm{Cr}_{0.33}\right)_{0.90} \mathrm{Fe}_{0.10}$ - coupe $(001)_{\mathrm{cfc}}$ (fluence $=5.10^{19} \mathrm{e}^{-/ \mathrm{cm}^{2}}$ ) (fluence $=8.10^{19} \mathrm{e}^{-/} / \mathrm{cm}^{2}$ )

Figure 2 : Diagrammes de diffraction des alliages $\left(\mathrm{Ni}_{0.67} \mathrm{Cr}_{0.33}\right)_{1-\mathrm{x}} \mathrm{Fe}_{\mathrm{x}}$ à la fluence maximum d'irradiation. 


\section{DISCUSSION}

L'irradiation accélère donc de façon importante la mise en ordre des alliages que nous étudions. Cet effet provient vraisemblablement de la sursaturation de défauts qui augmente la diffusion atomique. Nous avons donc évalué les concentrations de défauts présents.

A la température de nos irradiations les différentes espèces de défauts sont mobiles. Les dislocations étant les puits dominants, l'équation bilan des concentrations de défauts ponctuels s'écrit [12] :

$$
\frac{d c_{x}}{d t}=k_{0}-4 \pi r^{*}\left(D_{i}+D_{l}\right) c_{i} c_{l}-D_{x} \rho c_{x}
$$

où $\mathrm{x}$ est le type de défaut (i pour interstitiel, 1 pour lacune),

$c_{x}$ la concentration du défaut $x$,

$\mathrm{k}_{0}$ le taux de création de paires de Frenkel,

$\mathrm{r}^{*}$ le rayon de recombinaison,

$D_{x}$ le coefficient de diffusion du défaut $x$,

$\rho$ la densité de dislocations.

Un régime stationnaire s'établit, dans lequel la création de défauts compense la recombinaison et l'élimination sur les dislocations et les surfaces [13]. Le temps caractéristique d'établissement de cet équilibre et les populations de défauts à l'état stationnaire sont obtenus à partir des énergies de formation et de migration des défauts, de la densité de puits (faible, estimée à $10^{8} \mathrm{dislo} / \mathrm{cm}^{2}$ ) et d'une énergie seuil de déplacement de $24 \mathrm{eV}$ [14]. Nous avons utilisé les énergies obtenues par Dimitrov et al. [15][16], Berroudji [17] et par Huguenin et al. [18] pour le même alliage $\mathrm{Ni}_{0.73} \mathrm{Cr}_{0.18} \mathrm{Fe}_{0.09}$, relativement voisin des nôtres : $\mathrm{H}_{i}^{\mathrm{m}}=0,5 \mathrm{eV}$ [15] à $0,6 \mathrm{eV}[17], \mathrm{H}_{1}^{\mathrm{m}}=1,1 \mathrm{eV}$ [18] à $1,5 \mathrm{eV}$ [16] et $\mathrm{H}_{1}^{f}=1,78 \mathrm{eV}$ [16]. Le facteur prè-exponentiel pour les coefficients de diffusion des lacunes et des interstitiels est pris égal à 1 .

L'état stationnaire obtenu correspond à un régime de recombinaisons dominantes du fait de la faible concentration de puits. Il est atteint en quelques minutes et les concentrations à l'équilibre sont environ $\mathrm{c}=10^{-7}$ à $10^{-9}$ et $\mathrm{c}_{\mathrm{i}}<10^{-13}$. Le régime stationnaire est donc établi pratiquement depuis le début de l'irradiation, et le rôle de ces faibles concentrations de défauts sur la résistivité est négligeable. L'augmentation de résistivité observée en début d'irradiation est donc attribuée, comme dans le cas des recuits thermiques, à un accroissement de l'ordre à courte distance [8].

On a comparé sur la figure 3 l'évolution de la résistivité électrique de $\mathrm{Ni}_{0.67} \mathrm{Cr}_{0.33}$ en fonction du temps pour 2 types de vieillissement : un vieillissement thermique à $500^{\circ} \mathrm{C}$ et le vieillissement sous irradiation à $400^{\circ} \mathrm{C}$. Le plateau de résistivité est beaucoup plus court et la chute beaucoup plus rapide sous irradiation qu'en recuit thermique. La variation de résistivité estimée à saturation (donc l'état d'ordre final) semble aussi plus importante sous irradiation. Si l'on suppose que le principal effet de l'irradiation est d'accélérer la mise en ordre sans influer sur l'état d'équilibre final, il est normal d'obtenir à $400^{\circ} \mathrm{C}$ un état plus ordonné qu'à $500^{\circ} \mathrm{C}$.

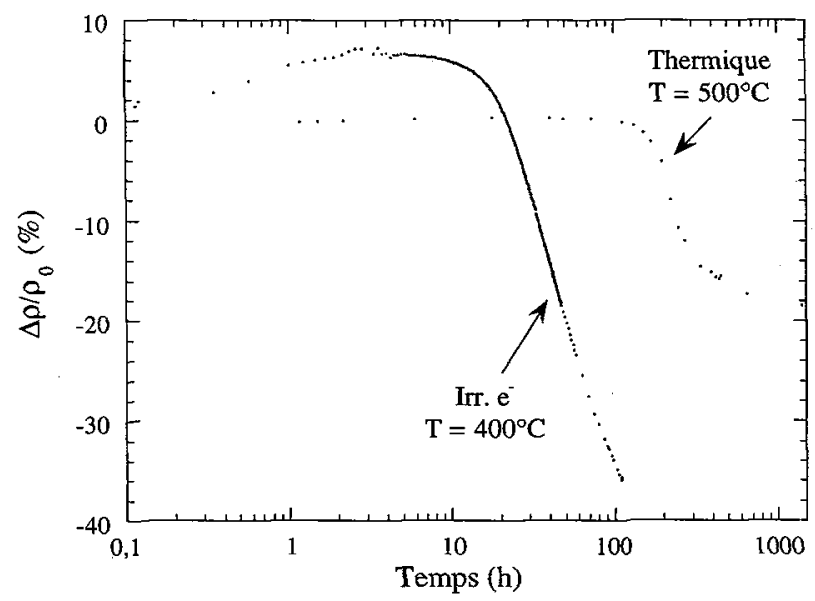

Figure 3 : Comparaison de l'évolution de la mise en ordre dans $\mathrm{Ni}_{0.67} \mathrm{Cr}_{0.33}$ par mesure de résistivité sous recuit isotherme et sous irradiation électronique. 
Une comparaison directe à la même température n'est pas possible actuellement car la mise en ordre par vieillissement thermique ne peut être étudiée qu' autour de $500^{\circ} \mathrm{C}$ (à plus haute température la force motrice est trop faible, et à plus basse température la mobilité trop réduite), et notre système de chauffage par le faisceau d'électrons ne nous permet pas pour l'instant d'irradier au-delà de $400^{\circ} \mathrm{C}$ en conservant une bonne homogénéité du dommage dans l'épaisseur de l'échantillon.

La présence de fer agit fortement sur l'évolution de la cinétique de mise en ordre sous irradiation comme le montre la figure 1. La chute de résistivité est plus tardive et plus faible lorsque l'alliage contient plus de fer. Cependant, la relation quantitative entre variation de résistivité et degré d'ordre n'est pas établie et n'a pas de raison d'être identique pour toutes les compositions.

Au contraire les diagrammes de diffraction d'électrons en fin d'irradiation semblent montrer que les échantillons présentent des taches de surstructure d'intensités voisines alors qu'ils ont des variations de résistivité très différentes.

Pour évaluer le degré d'ordre atteint, nous avons analysé nos cinétiques (hors la montée initiale de résistivité), en utilisant le modèle simple de Dienes [19]. La mise en ordre est décrite par une réaction chimique qui représente l'échange direct de paires de premiers voisins sur les différents sous-réseaux de la surstructure.

L'équation cinétique pour le paramètre d'ordre à longue distance $S$ de notre composé $\mathrm{AB}_{2}$ s'écrit :

$$
\frac{\mathrm{dS}}{\mathrm{dt}}=\mathrm{ve}^{-\mathrm{E}_{0} / \mathrm{kT}}\left[\frac{2}{9}(1-\mathrm{S})^{2}-\mathrm{e}^{-\mathrm{V}_{0} \mathrm{~S} / \mathrm{kT}}\left\{\mathrm{S}+\frac{2}{9}(1-\mathrm{S})^{2}\right\}\right]
$$

où $v$ est une fréquence d'attaque, $\mathrm{E}_{0}$ une énergie de barrière et $\mathrm{V}_{0}$ l'énergie gagnée par mise en ordre.

La température de transition expérimentale $\mathrm{T}_{\mathrm{c}}$, d'environ $580^{\circ} \mathrm{C}$ pour $\mathrm{Ni} 2 \mathrm{Cr}$, fixe le paramètre $\mathrm{V}_{0}$ de ce modèle $\left(\mathrm{V}_{0} / \mathrm{k}=3753.6 \mathrm{~K}\right)$. De façon arbitraire, ne connaissant pas $\mathrm{T}_{\mathrm{c}}$ pour les autres compositions, nous avons supposé que $\mathrm{V}_{0}$ ne varie pas avec la concentration de fer.

D'autre part, nous avons admis avec Muto [20] que la résistivité suit en fonction du paramètre d'ordre une loi du type $\rho=\left(\rho_{o}-\rho_{d}\right) S^{2}+\rho_{d}$, où $\rho_{d}$ est la résistivité de l'alliage complètement désordonné, et $\rho_{o}$ celle de l'alliage parfaitement ordonné. Enfin, dans ce modèle dS/dt est nul pour $S=0$ et la mise en ordre ne peut démarrer que s'il y a un ordre initial (faible) $S_{\mathrm{i}}$ au temps 0 . C'est un paramètre supplémentaire du modèle.

Dans le tableau 1 sont portés les paramètres $\Delta \rho_{o-d}=\rho_{o}-\rho_{d}, \beta=v e^{-\mathrm{E}_{\mathrm{o}} / \mathrm{kT}}$ et $\mathrm{S}_{\mathrm{i}}$ après ajustement sur les irradiations à $400^{\circ} \mathrm{C}$. A cette température, la valeur à saturation du paramètre d'ordre est $S_{\text {sat }}=0,791$ pour les 3 alliages. En figure 4, le paramètre d'ordre à longue distance $S$ est tracé en fonction du temps d'irradiation.

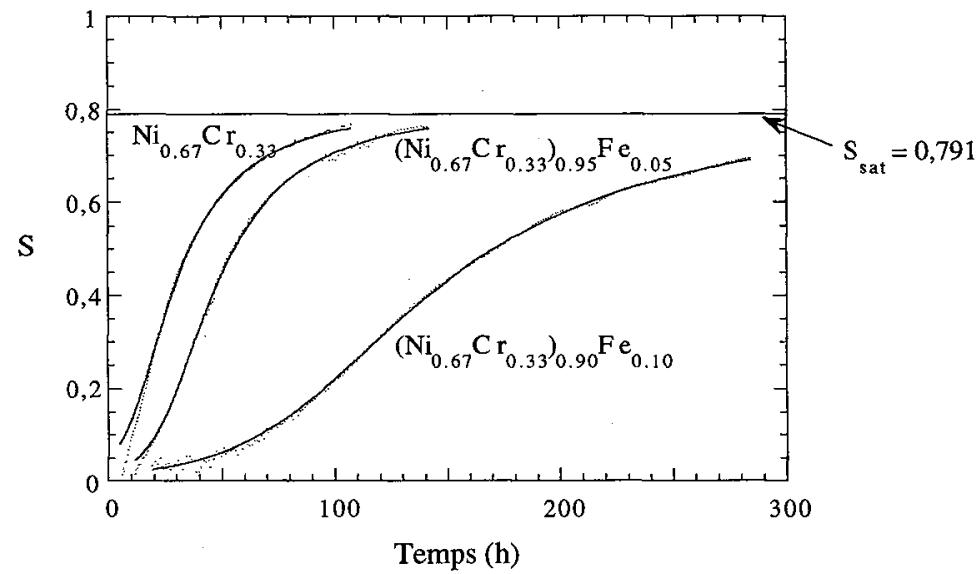

Figure 4 : Evolution du paramètre d'ordre $S$ en fonction du temps d'après le modèle de Dienes [19] dans $\left(\mathrm{Ni}_{0.67} \mathrm{Cr}_{0.33}\right)_{1-\mathrm{x}} \mathrm{Fe}_{\mathrm{x}}$ à $400^{\circ} \mathrm{C}$. 


\begin{tabular}{|c|c|c|c|}
\cline { 2 - 4 } \multicolumn{1}{c|}{} & $\mathrm{Ni}_{2} \mathrm{Cr}$ & $\left(\mathrm{Ni}_{0.67} \mathrm{Cr}_{0.33}\right)_{0.95} \mathrm{Fe}_{0.05}$ & $\left(\mathrm{Ni}_{0.67} \mathrm{Cr}_{0.33}\right)_{0.90} \mathrm{Fe}_{0.10}$ \\
\hline$\Delta \rho_{0-\mathrm{d}}(\mu \Omega-\mathrm{cm})$ & 116 & 65 & 44 \\
\hline$\beta\left(10^{-5} \mathrm{~s}^{-1}\right)$ & 13,6 & 11,5 & 3,8 \\
\hline $\mathrm{S}_{\mathrm{i}}$ & 0,059 & 0,015 & 0,013 \\
\hline
\end{tabular}

Tableau 1 : Paramètres ajustés pour les alliages $\left(\mathrm{Ni}_{0.67} \mathrm{Cr}_{0.33}\right)_{1-\mathrm{x}} \mathrm{Fe}_{\mathrm{X}}$ à $400^{\circ} \mathrm{C}$.

D'après le modèle, les 3 alliages atteignent en fin d'irradiation des degrés d'ordre très voisins (de 0,69 à 0,76 ) alors que leurs variations de résistivité sont très différentes (voir figure 1). Toutefois, ce modèle simple ne tient pas compte du rôle des lacunes dans la mise en ordre et de la présence du fer, et pratiquement pas de la structure atomique de l'alliage. Des modèles plus sophistiqués seraient nécessaires pour expliquer complètement les cinétiques observées.

\section{CONCLUSION}

L'irradiation accélère fortement la mise en ordre de type $\mathrm{Pt}_{2} \mathrm{Mo}$ des alliages $\mathrm{Ni}_{0.67} \mathrm{Cr}_{0.33}$, $\left(\mathrm{Ni}_{0.67} \mathrm{Cr}_{0.33}\right)_{0.95} \mathrm{Fe}_{0.05}$ et $\left(\mathrm{Ni}_{0.67} \mathrm{Cr}_{0.33}\right)_{0.90} \mathrm{Fe}_{0.10}$. Ce dernier $\mathrm{n}$ 'a jamais pu être ordonné thermiquement. Les cinétiques suivies à $400^{\circ} \mathrm{C}$ par résistivité sont similaires à celles obtenues lors du vieillissement thermique de $\mathrm{Ni}_{2} \mathrm{Cr}$ à $500^{\circ} \mathrm{C}$; en particulier elles présentent un "état $\mathrm{K}$ ". La présence de fer augmente, sous irradiation, la durée du plateau de l'état $\mathrm{K}$ et ralentit fortement la réaction de mise en ordre à longue distance.

\section{Remerciements}

Les auteurs remercient Didier Régen et René Caudron (OM-ONERA, Châtillon) pour la fabrication des alliages et pour le vieillissement thermique.

\section{Bibliographie}

[1] Thomas J. H. et Davies R. M., Phil. Mag. 22 (1936) 681.

[2] Taylor A. et Hinton K.G., J. Inst. Metals 81 (1952-1953) 169.

[3] Baer H. G., Naturwiss. 43 (1956) 298.

[4] Gomankov V. I., Litvine D. F., Lochmanov A. A. et Lyachenko B. G., Fiz. Metallov i Metalloved. 14 (1962) 305.

[5] Coulomb P., Reynaud F. et Lasserre A., C. R. Acad. Sc. Paris 269 (1969) 404-407.

[6] Vintaykin Y. Z. et Urushadze G. G., Fiz. Metallov i Metalloved. 27 (1969) 132-141.

[7] Nordheim R. et Grant N. J., J. Inst. of Metals 82 (1953-1954) 440-444.

[8] Hirabayashi M. et al., Trans. J.I.M. 10 (1969) 365-371.

[9] Taunt R. J. et Ralph B., Phys. stat. sol. (a) 29 (1975) 431-442.

[10] Van Duysen J.C. Rapport interne EDF HT-41/NEQ 1117-A (1990).

[11] Rivière J. P. et Eymery P., Radiation Effects 37 (1978) 155-160.

[12] Barbu A. et Martin G., Solid State Phenomena 30 \& 31 (1993) 179-228.

[13] Sizmann R., Journal of Nuclear Materials 69 \& 70 (1978) 386-412.

[14] mesure d'énergie seuil dans $\mathrm{Ni}_{2} \mathrm{Cr}$, non publié.

[15] Dimitrov C., Huguenin D., Moser P. et Dimitrov O., Journal of Nuclear Materials 174 (1990) 22-34.

[16] Dimitrov C. et Dimitrov O., Journal of Nuclear Materials 152 (1988) 21-29.

[17] Berroudji A., Thèse, Université de Grenoble I (1984).

[18] Huguenin D. et al., Mater. Sci. Forum 15 - 18 (1987) 1269.

[19] Dienes G., J. Acta Met. 3 (1955) 549-557.

[20] Muto T. et Takagi Y., Solid State Physics Vol. I (Seitz F. et Turnbull D. Eds., Academic Press Inc., New York, 1955) pp. 193-282. 